\title{
Histological Changes in Congenital and Acquired Blepharoptosis
}

\author{
FRANCIS C. SUTULA \\ Boston USA
}

\begin{abstract}
Summary
Histological specimens of eyelids from patients with congenital genuine ptosis and acquired involutional ptosis are compared. In congenital ptosis there is evidence of true muscular dystrophy of the levator muscle as demonstrated by: loss of cross striations, random decrease in muscle fibre diameter, sarcolemmal retraction, nuclei alignment, and fibrous and fatty tissue displacement of the striated fibres.

In involutional ptosis disinsertion of the levator aponeurosis from its normal insertion on the inferior-anterior surface of the tarsus is demonstrated. Correlation between clinical presentation and surgical correction is explained in light of these findings.
\end{abstract}

Patients with ptotic eyelids have been classified as 'congenital' or 'acquired' based on their history and clinical manifestations. This division has been extremely useful since empirical evidence has indicated that different techniques in management are necessary.

Histological examination has clarified the nature of these conditions and, therefore, their clinical manifestations. Also, examination of the cause indicates an appropriate approach to the treatment options available.

By definition, congenital ptosis presupposes onset at birth. However, most authorities prefer, for practical reasons, to eliminate patients whose ptosis is associated with birth trauma, synkinetic movements, and major malformations from this 'congenital' classification. Therefore, 'congenital' genuine ptosis is a specific subcategory of this first designation. Additionally, the exact onset of the ptosis is somewhat difficult to determine, Isaksson $^{1}$ cites five year maternity records from Sweden in which no recording of ptosis was recorded in the perinatal period. However, it appears that the onset is usually in early infancy and the course is often characterised by little change in either the amount of the ptosis or the function of the levator muscle. Patients who present at an older age will describe a gradual progression of the ptosis over a period of time. Occasionally, a history will be obtained wherein ptotic eyelids improved spontaneously during the first year of life.

A characteristic of both 'congenital' and involutional ptosis is that the upper eyelid crease and upper eyelid fold may be abnormally formed. The crease may be asymmetrical with the contralateral side; there may be duplication of the crease or there may be an entire absence of the crease. In 'congenital' ptosis, however, the amount of levator function correlates directly with the amount of the ptosis. (Fig. 1) Absence of the upper eyelid crease signifies no levator function. (Fig. 2) Almost invariably the ptotic eyelid is higher than the normal eyelid in downgaze (Fig. 3) ('almost the sine qua non'). The exception to this rule is trauma, either accidentally or surgically produced.

The classification of acquired ptosis

Correspondence to: Francis C. Sutula, MD, 3 Hawthorne Place \#106, Boston, MA 02114, USA.

From the Department of Ophthalmology, Harvard Medical School and Massachusetts Eye and Ear Infirmary, Boston, USA. 


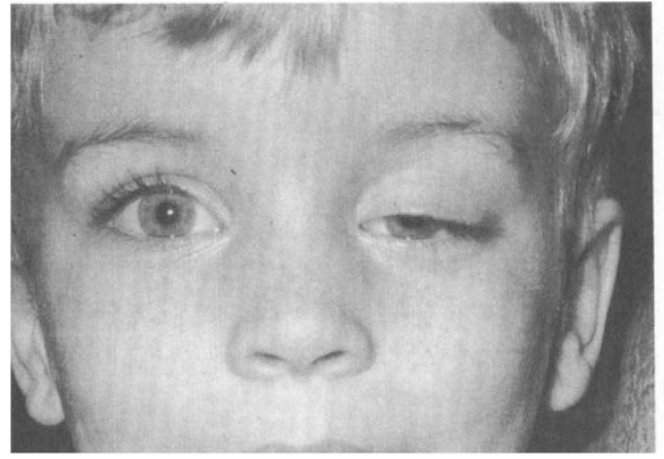

Fig. 1A. View of the patient in the primary position. The right eye is at a normal level and the left eye has a five millimetre ptosis.

includes specific aetiologies determined by history and examination. The classification includes specific causes as well as non-specific grouping. In all acquired cases, however, a

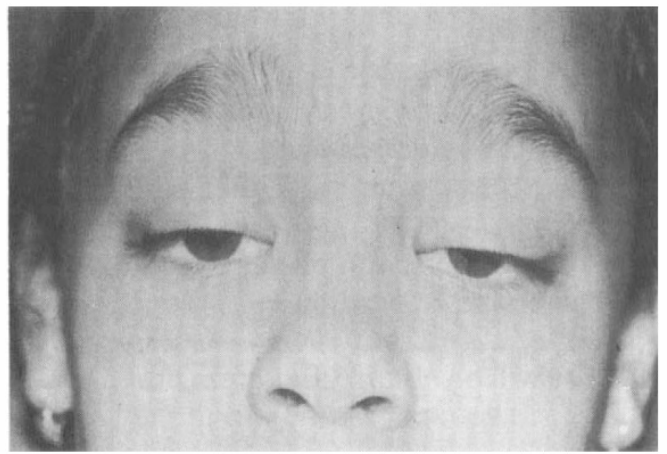

Fig. 2. Absence of the upper eyelid crease. Notice brow position and shape.

time may be specified during which the ptosis became apparent. Previous to this the eyelid functioned normally. The patient usually has photographs taken at different times so that a

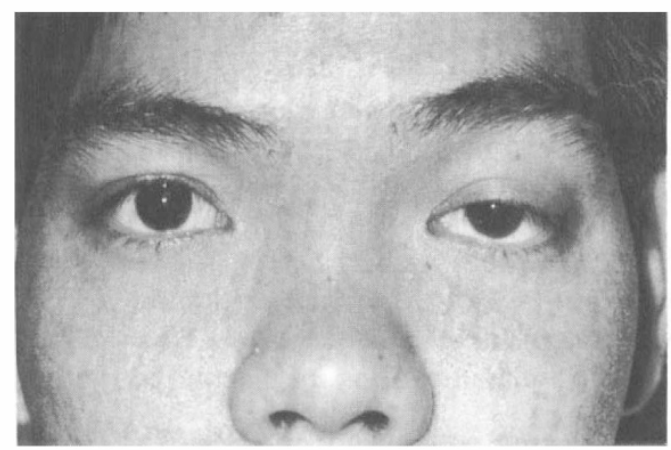

Fig. 3A. View of a patient with congenital ptosis in primary gaze.

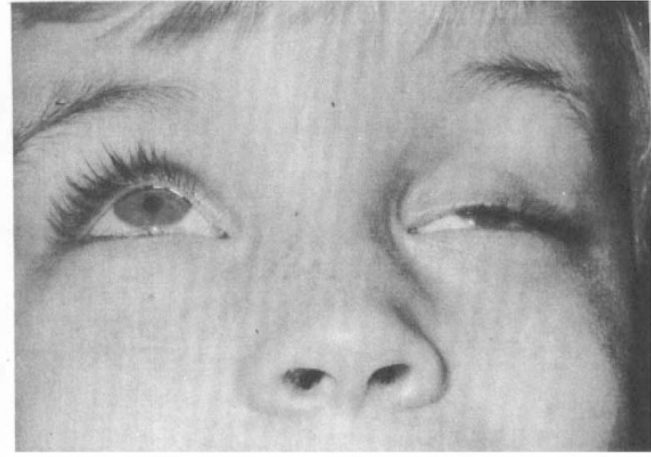

Fig. 1B. In upgaze there is normal excursion on the right and essentially no elevation on the left.

time scale of progression can be charted. There are often precipitating factors such as a history of trauma, intraocular surgery, or associated systemic diseases.

The specific classifications include traumatic, mechanical, neurogenic, and myogenic aetiologies. The histological picture coincides with the classification. Focal cicatrisation occurs in trauma cases; motoneuronal and myogenic degeneration occurs in their respective groups.

The involutional type of acquired ptosis has, in the past, been very loosely classified and it was often incorporated as a part of the myogenic listing. The function of the levator muscle in the involutional type of ptosis usually demonstrates a normal or near normal level. This normal excursion of the upper eyelid is often associated with extreme levels of ptosis. (Fig. 4) The upper eyelid crease, may be duplicated or may be higher in the upper eyelid sulcus than the contralateral side. (Fig. 5) Clinicians have repeatedly observed while

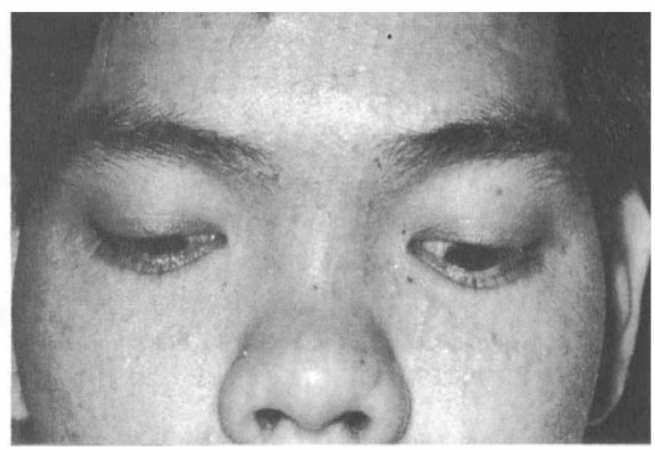

Fig. 3B. In downgaze demonstrating the lid lag of the ptotic eyelid. 
performing the surgical repair of these patients, that the required resection of the levator was much less than that needed for 'congenital' genuine ptosis. The amount of resection was determined empirically. In congenital ptosis, the range of levator resections was recommended to be between 14 and $28 \mathrm{~mm}$. In the acquired variety, the range was limited to 10 to $15 \mathrm{~mm}^{2}$. Also, in the acquired variety, many procedures unrelated to the levator muscle were recommended. These included: excising the tarsus and the conjunctiva with or without excision of fibres from Mueller's muscle ${ }^{3}$ (the Fasanella-Servat procedure) or resection of Mueller's muscle and conjunctiva after pharmacological evaluation showed a satisfactory result.

The first recorded histological examination of a patient with congenital ptosis was in 1879 by Heuck. ${ }^{4} \mathrm{He}$ showed a postmortem specimen in which there was degeneration and fibrosis in the levator muscle complex. Other authors reported similar findings. However, Berke and Wadsworth in $1955^{5}$ reported their
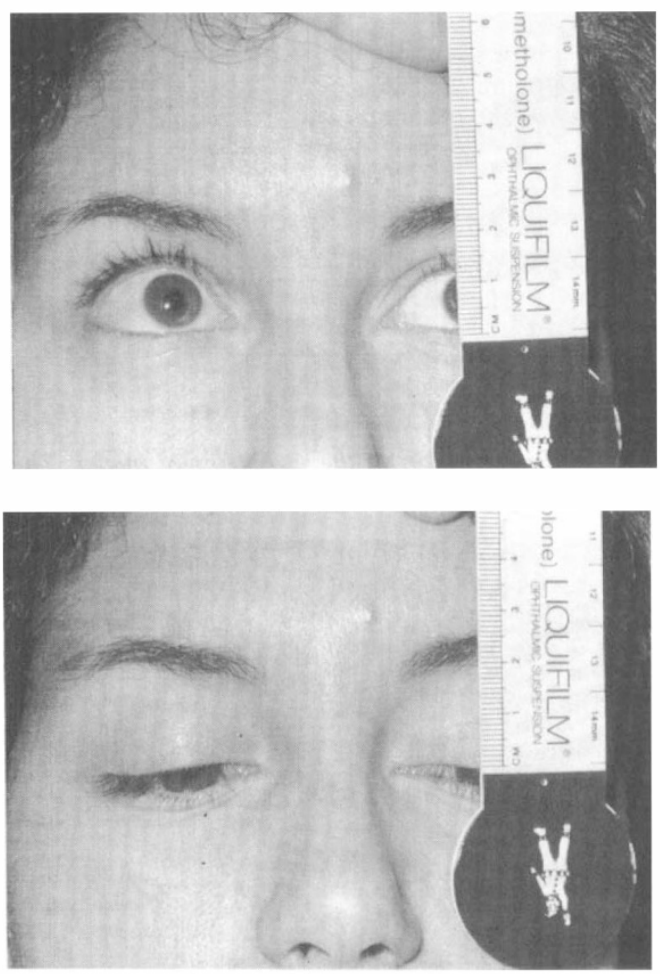

Figs 4A \& B. Demonstrates the normal upper eyelid excursion from downgaze to left gaze. findings from 82 specimens with ptosis. Of these specimens, 44 were of the 'congenital' genuine category. They demonstrated the following facts:

(1) Mueller's muscle was present in both the acquired and congenital types of ptosis.

(2) Striated fibres were always found in the levator muscle in acquired ptosis, birth trauma ptosis and synkinetic ptosis.

(3) Striated fibres were always found in patients with ptosis of $2 \mathrm{~mm}$ or less.

(4) Striated fibres were found in 54 per cent of patients with ptosis of $3 \mathrm{~mm}$.

(5) Striated fibres were not present in patients with ptosis of 4 or more mm.

Berke later commented that the amount of ptosis and the amount of levator function directly correlated and therefore, was associated with the number of striated fibres present in the muscle.

Isaksson ${ }^{6}$ reported in 1962, his studies of 48 specimens with ptosis. He also correlated the number of specimens wherein striated fibres were observed. The number of specimens with fibres decreased dramatically in the severe forms of ptosis.

The histological findings described by Berke and Wadsworth are compatible with muscular dystrophic conditions. The muscle fibres became atrophic, pale and flabby. There was decrease in the cross-sectional diameter of the muscle fibres (Fig. 6) associated with hypertrophy and pseudohypertrophy in the same area. Hyalin degeneration and vacuolisation was seen. Shrinkage from the sarcolemmal sheet (Fig. 7) and prolifera-

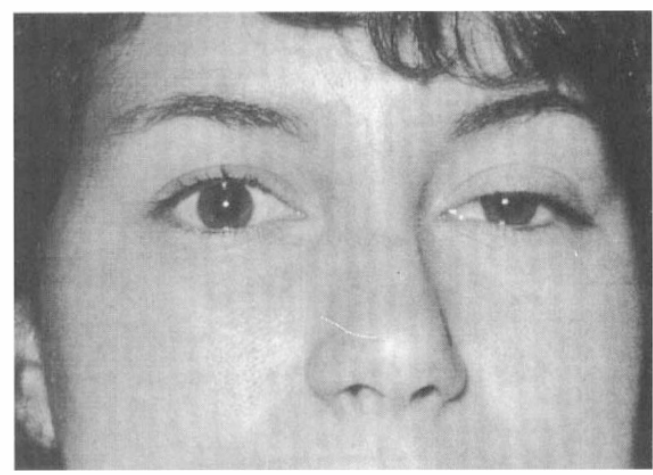

Fig. 5. The same patient in primary position showing the higher upper eyelid crease on the left. 
tion of the sarcolemmal nuclei (Fig. 8) was also seen. The intrafusal muscle fibres of muscle spindles were preserved after large portions of the muscle disappeared. There was an increase in the endomysial collagen (fibrous tissue in the muscular septa and fat deposition within the muscle fibres). (Fig. 9) Of course, there was loss of cross striations and, associated with muscle death, a slight inflammatory reaction. These findings differ from those which normally appear with disuse, vascular, or denervation degeneration. In these conditions, the atrophic muscle fibres are found lying beside normal fibres belonging to a different motoneuronal group. There is also brown atrophy (perinuclear lipochrome pigment deposition) associated with neuronal degeneration.

These findings suggest that so-called 'congenital' ptosis is a muscuar dystrophy of obscure origin. Muscular dystrophies are often associated with specific muscle groupsfor example, the limb girdle dystrophy of Duchenne. There is often a genetic pre-

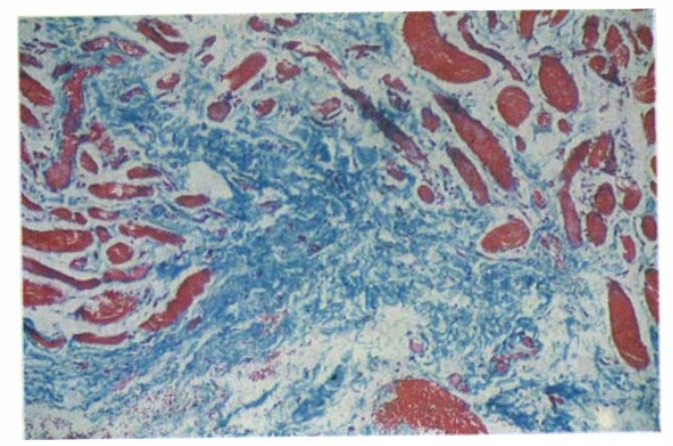

Fig. 6. Showing the variation in the muscle fibre size and fibrous tissue replacement of the muscular bundles.

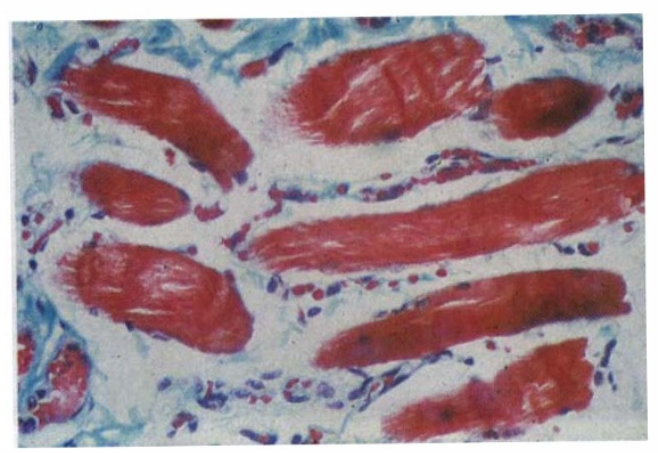

Fig. 7. Shows shrinkage from the sarcolemmal sheet. disposition for muscular dystrophies. In 'congenital' ptosis, especially the most severe form blepharophimosis syndrome, there are also identifiable hereditary patterns.

The acquired ptosis of the involutional type is a relatively newly described entity. This ptosis was first reported by Jones, Quickert and Paris ${ }^{7}$ based on anatomical dissections performed by Lester Jones. Marvin Quickert ${ }^{8}$ reported that repair of the levator aponeurosis defect could achieve a satisfactory postoperative result. This was the first departure from the empirical, i.e. 'cookbook,' methods which had earlier been the hallmark of surgery for acquired ptosis. In 1978 Dortzbach and Sutula ${ }^{9}$ histologically examined patients with this type of acquired ptosis. These patients often have moderate to severe ptosis with good levator function and high upper eyelid creases (Table I). Nineteen eyelids were examined. Two of the specimens were unsuitable and 17 were satisfactory (Table II). All but 2 of these specimens

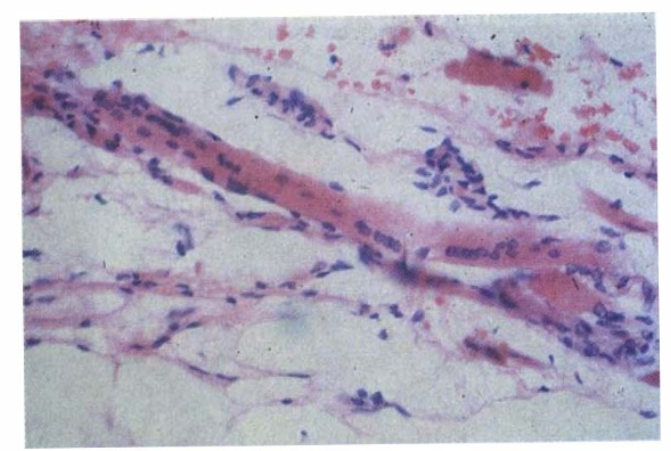

Fig. 8. Proliferation of the sarcolemmal nuclei. The nuclei are lining up along the edge of the muscle fibre.

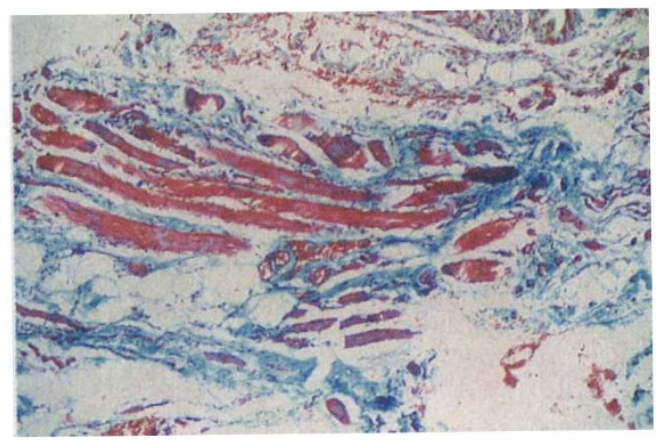

Fig. 9. Fibrous tissue and fatty changes along muscle fibres. 
showed disinsertion of the levator aponeurosis (Fig. 10). Mueller's muscle was found to be present and normal in all cases. The levator aponeurosis was found to be normal in the specimens examined.*

The surgical repair consisted of reattaching what was identified as the distal end of the

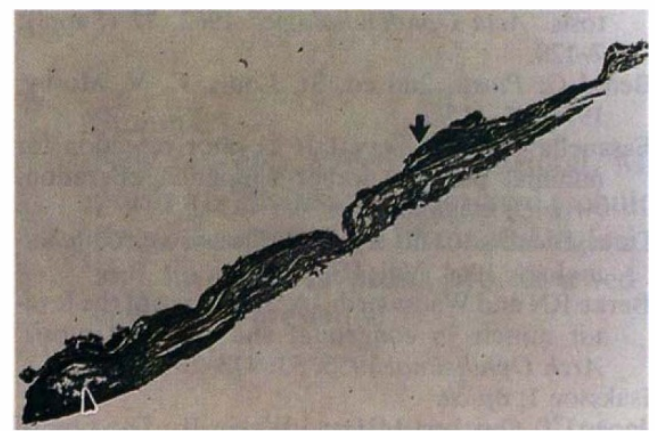

Fig. 10. The superior portion of the tarsus is in the lower left hand corner. The distal end of the levator aponeurosis is in the upper right hand corner. In between there is loose connective tissue and Mueller's muscle.

( ${ }^{*}$ Author's note: While that was true in the original study of 19 eyelids, more recent examinations have shown specimens of the levator muscle to demonstrate myogenic degeneration.)

Table III. Involutional blepharoptosis patient data levator aponeurosis to its normal insertion site- the inferior-anterior surface of the tarsus. The vast majority of these patients produced excellent results (Table III). Furthermore, selected levator biopsies in patients meeting these criteria have shown a high proportion of striated muscular content. Therefore, confirmation of the entity of

Table I. Characteristics of involutional blepharoptosis of the aponeurogenic type

1. Acquired

2. Older age group

3. Possible precipitating factors
a. intraocular surgery
b. ocular inflammation
c. topical steroids

4. Good levator function (usually $12 \mathrm{~mm}$ or greater)

5. High upper eyelid crease

6. Thin upper eyelid (occasionally)

Table II. Histopathological results

\begin{tabular}{lr} 
Eyelids with satisfactory biopsies & 17 \\
Eyelids with disinsertions & $12(71 \%)$ \\
Eyelids with attenuations & $2(12 \%)$ \\
Eyelids with inconclusive specimens & $3(17 \%)$ \\
& \\
\hline
\end{tabular}

\begin{tabular}{cccccc}
\hline No. eyelids & Patient & Eyelid & $\begin{array}{c}\text { Gross anatomical } \\
\text { defect }(\text { O.R.) }\end{array}$ & $\begin{array}{c}\text { Histological } \\
\text { defect }\end{array}$ & $\begin{array}{c}\text { Surgical } \\
\text { results }\end{array}$ \\
\hline 1 & A & RT & YES & YES & SI \\
2 & A & LT & YES & S.U. & SI \\
3 & B & RT & UND & YES & MI $^{2}$ \\
4 & B & LT & UND & YES & SI $^{2}$ \\
5 & C & RT & NO & ATTEN. & II $^{2}$ \\
6 & C & LT & NO & INCL. & SI \\
7 & D & LT & YES & YES & MI \\
8 & E & RT & YES & ATTEN. & SI \\
9 & E & LT & YES & YES & SI \\
10 & F & RT & YES & YES & SI \\
11 & F & LT & YES & YES & SI \\
12 & G & RT & YES & YES & SI \\
13 & H & RT & YES & YES & SI \\
14 & H & LT & YES & YES & SI \\
15 & I & RT & YES & YES & SI \\
16 & I & LT & YES & INCL. & S \\
17 & J & RT & YES & YES & SI \\
18 & J & LT & YES & INCL. & YES \\
19 & K & LT & YES & & \\
\hline
\end{tabular}

1 = Attenuation; 2 = Levator tuck; S.U. = specimen unsatisfactory; Incl $=$ inconclusive $;$ SI $=$ satisfactory improvement; $\mathrm{MI}=$ minimal improvement; Und = undetermined. 
aponeurosis disinsertion as a common type of acquired ptosis indicates that amelioration of the condition can be accomplished by reapproximating the normal anatomy rather than utilising various manipulations of uninvolved tissue in order to shorten the eyelid.

In conclusion, what we call 'congenital' genuine ptosis is, in fact, considered a muscular dystrophy as demonstrated by various degrees of muscular degeneration. One point of contention, however, is that 'dystrophy,' implies normal development followed by degeneration. It is likely that we are dealing with an abnormal development, i.e. dysgenesis. This is supported by the fact that levator muscle develops at a later time and individually from the other extraocular muscle. The clinical picture of congenital ptosis correlates with the relative amount of striated tissue present. The amount directly relates to the upper eyelid excursion, the formation of the eyelid crease, as well as the presence of lid lag in downgaze.

The involutional type of acquired ptosis is primarily of aponeurosis origin. It often shows normal muscular function and a superior displacement of the upper eyelid crease second- ary to the disinsertion and high position of the distal levator aponeurosis.

The author would like to thank Victor Elner MD for preparation of the photographs and to Marlene Ingram for her assistance in compiling the manuscript.

\section{References}

${ }^{1}$ Isaksson I: Studies on congenital genuine blepharoptosis. Acta Ophthalmologica 1962, 72 (Suppl): 7-120.

${ }^{2}$ Beard C: Ptosis. 2nd ed. St. Louis, C. V. Mosby, 1976: 91-115.

${ }^{3}$ Fasanella RM and Servat J: Levator resection for minimal ptosis: another simplified operation. Arch Ophthalmol 1961, 65: 493-6.

${ }^{4}$ Duke-Elder S: The eyelids. System of Ophthalmology. 1964. Vol. III. p. 898.

${ }^{5}$ Berke RN and Wadsworth JA: Histology of the levator muscle in congenital and acquired ptosis. Arch Ophthalmol 1955, 53: 413-28

${ }^{6}$ Isaksson I: op cit.

${ }^{7}$ Jones LT, Quickert MH and Wobig JL: The care of ptosis by aponeurosis repair. Arch Ophthalmol 1975, 93: 629-34.

${ }^{8}$ Paris GL and Quicker MH: Disinsertion of the aponeurosis of the levator palpebrae superioris muscle after cataract extraction. Am J Ophthalmol 1976, 81: 337-40.

${ }^{9}$ Dortzbach RK and Sutula FC: Involutional Blepharoptosis: A Histopathological Study. Arch Ophthalmol 1980, 98: 2045-9. 